\title{
Star Formation and Chemical Evolution of Damped Ly- $\alpha$ Systems
}

\author{
Jun Ma \\ Beijing Astronomical Observatory and Beijing Astrophysics Center \\ (BAC), National Astronomical Observatories, Chinese Academy of \\ Sciences, Beijing, 100012, P. R. China
}

\begin{abstract}
In this paper, we investigate the star formation and chemical evolution of damped Lyman- $\alpha$ systems (DLAs) based on the disk galaxy formation model developed by H. J. Mo, S. Mao, \& S. D. M. White (1998, MNRAS, 295, 319). We propose that the DLAs are the central galaxies of less massive dark haloes present at redshifts $z \sim 3$, and that they should inhabit haloes of moderately low circular velocity. We adopt the empirical Schmidt law of star formation rates, and a closed box model of chemical evolution in which an approximation known as instantaneous recycling is assumed. In calculating the predicted distribution of metallicities for DLAs in our models, two cases are considered. One is that, using the closed box model, empirical Schmidt law, and star formation epoch, the distribution of metallicity can be directly calculated. The other is that, when the simple gravitational instability of a thin isothermal gas disk first discussed by A. Toomre (1964, ApJ, 139, 1217) is considered, star formation occurs only in the region where the surface density of gas satisfies the critical value - rather than everywhere in the gas disk. We first obtain the region where star formation can occur by assuming that the disk has a flat rotation curve and that the rotational velocity is equal to the circular velocity of the surrounding dark matter halo. We then calculate the metallicity distribution for case one. We assume that star formation in each DLA lasts for a period of 1 Gyr from redshifts $z=3$. There is only one output parameter in our model, i.e., the stellar yield, which relates to the epoch of star formation. It is obtained by normalizing the predicted distribution of metallicity to the mean value of $1 / 13 Z_{\odot}$ as presented by M. Pettini, L. J. Smith, D. L. Kind, \& R. W. Hunstead (1997, ApJ, 486, 665). The predicted metallicity distribution is consistent with current (rather limited) observational data. A random distribution of galactic disks is taken into account.
\end{abstract}

